\title{
BMJ Open Medication-related experiences of patients with polypharmacy: a systematic review of qualitative studies
}

\author{
Christian Ulrich Eriksen (10 , ${ }^{1}$ Stavros Kyriakidis, ${ }^{1}$ Line Due Christensen, ${ }^{2}$ \\ Ramune Jacobsen, ${ }^{3}$ Jannie Laursen, ${ }^{4}$ Mikkel Bring Christensen, ${ }^{5}$ Anne Frølich ${ }^{6,7}$
}

To cite: Eriksen CU, Kyriakidis S, Christensen LD, et al.

Medication-related experiences of patients with polypharmacy: a systematic review of qualitative studies. BMJ Open 2020;10:e036158. doi:10.1136/ bmjopen-2019-036158

- Prepublication history and additional material for this paper are available online. To view these files, please visit the journal online (http://dx.doi. org/10.1136/bmjopen-2019036158).

$\mathrm{MBC}$ and $\mathrm{AF}$ contributed equally.

$\mathrm{MBC}$ and $\mathrm{AF}$ are joint senior authors.

Received 03 December 2019 Revised 02 July 2020 Accepted 08 July 2020

Check for updates

(c) Author(s) (or their employer(s)) 2020. Re-use permitted under CC BY-NC. No commercial re-use. See rights and permissions. Published by BMJ.

For numbered affiliations see end of article.

Correspondence to Christian Ulrich Eriksen; cueriksen@gmail.com

\section{ABSTRACT}

Background We aimed to synthesise qualitative studies exploring medication-related experiences of polypharmacy among patients with multimorbidity.

Methods We systematically searched PubMed, Embase and Cumulative Index to Nursing and Allied Health Literature in February 2020 for primary, peer-reviewed qualitative studies about multimorbid patients' medicationrelated experiences with polypharmacy, defined as the use of four or more medications. Identified studies were appraised for methodological quality by applying the Critical Appraisal Skills Programme checklist for qualitative research, and data were extracted and synthesised by the meta-aggregation approach.

Results We included 13 qualitative studies, representing 499 patients with polypharmacy and a wide range of chronic conditions. Overall, most Critical Appraisal Skills Programme items were reported in the studies. We extracted 140 findings, synthesised these into 17 categories, and developed five interrelated syntheses: (1) patients with polypharmacy are a heterogeneous group in terms of needing and appraising medication information; (2) patients are aware of the importance of medication adherence, but it is difficult to achieve; (3) decision-making about medications is complex; (4) multiple relational factors affect communication between patients and physicians, and these factors can prevent patients from disclosing important information; and (5) polypharmacy affects patients' lives and self-perception, and challenges with polypharmacy are not limited to practical issues of medication-taking.

Discussion Polypharmacy poses many challenges to patients, which have a negative impact on quality of life and adherence. Thus, when dealing with polypharmacy patients, it is crucial that healthcare professionals actively solicit individual patients' perspectives on challenges related to polypharmacy. Based on the reported experiences, we recommend that healthcare professionals upscale communicative efforts and involve patients' social network on an individualised basis to facilitate shared decision-making and treatment adherence in multimorbidpatients with polypharmacy.

\section{INTRODUCTION}

Patients with multimorbidity often have complex treatment regimens requiring them to take several medications concurrently,

\section{Strengths and limitations of this study}

- We employed a stringent process of selecting and appraising studies.

- The credibility of our results is enhanced by triangulation of findings from different studies.

- The transferability of our findings to all patients taking more than four medications is unknown.

- A single author completed data extraction, potentially limiting the confirmability of our study.

a phenomenon termed polypharmacy. ${ }^{1}$ Although a wide range of definitions exist for polypharmacy, a common definition is simultaneous use of five or more medications. ${ }^{2}$ Polypharmacy is often clinically appropriate and is thus not necessarily a sign of inappropriate care, ${ }^{3}$ but the use of multiple medications in the elderly is associated with increases in mortality, adverse drug events, falls, length of hospital stay, and readmission rates. ${ }^{2}$ In addition, a high frequency of medication errors has been reported among primary care patients with polypharmacy, ${ }^{45}$ and discrepancies often exist between medication lists in the primary and secondary healthcare sectors. ${ }^{5-7}$ A 2018 systematic review found that 26 of 33 studies examining patient-related risk factors for medication errors reported positive associations between polypharmacy and medication errors. ${ }^{8}$

High rates of non-adherence have been identified among newly discharged patients with polypharmacy, and an increased number of medications prescribed in hospitals is associated with non-adherence. ${ }^{9}$ In general, medication-related burden and patient experiences of medications negatively affect patients' health and well-being, as well as their medication-related beliefs and behaviours. ${ }^{10}$ This can lead to non-adherence, poorer therapeutic outcomes, and patients independently altering their medications or continuing treatment, which negatively 
affects their well-being. ${ }^{10}$ Multiple studies demonstrate that most patients are interested in having one or more medications deprescribed if possible. ${ }^{11-17}$

Increasing numbers of people live with multimorbidity and require multiple medications, and an investigation of their experiences is essential to understand the phenomenon of polypharmacy. In addition, it is uncertain whether interventions to improve appropriate polypharmacy have clinically significant effects. ${ }^{18}$ Insight into medication-related experiences, defined as the sum of events involving drug therapy that a patient has in their lifetime, ${ }^{19}$ of multimorbid patients with polypharmacy can help identify opportunities to improve care, develop effective interventions and align research with what matters most to patients. The aim of this study was to explore medication-related experiences of polypharmacy among patients with multimorbidity through a systematic review of qualitative studies.

\section{METHODS}

We conducted a preplanned structured search of qualitative studies exploring medication-related experiences among multimorbid patients with polypharmacy. Although many studies defined polypharmacy as five or more medications, some defined it as four or more medications. ${ }^{2}$ To reflect varying definitions and capture all relevant studies, we used the latter definition. A systematic review of qualitative studies is ideal for our purpose because it provides an overview and reinterpretation of existing evidence from qualitative research. ${ }^{20}$

\section{Search strategy}

We identified search terms relevant to the three major concepts of our study aim: patient experiences, polypharmacy and qualitative research. Synonyms and search terms were identified by reviewing the protocol for a previous review of qualitative studies on medicationrelated burden (not focused on patients with polypharmacy),${ }^{10}$ as well as other qualitative review protocols. We also conducted a simple search in the PubMed database to identify relevant terms and medical subject headings. We applied the search strategy to PubMed, Embase and Cumulative Index to Nursing and Allied Health Literature (CINAHL) in February 2020 (online supplementary table 1). Searching PubMed and CINAHL in combination is recommended for identifying qualitative studies. ${ }^{21}$ We modified the search strategy to specific database requirements, tailoring the use of indexing terms or symbols for wildcards. Search filters were not applied. We also screened the reference lists of included studies and relevant systematic reviews for additional studies. Finally, we removed duplicate references.

To be eligible for inclusion, studies had to (1) be peerreviewed research concerning medication-related experiences; (2) include patients with multimorbidity and polypharmacy aged 18 years or older taking four or more medications; (3) use a qualitative methodology; (4) be reported in English (or Danish, Swedish or Norwegian for practical reasons); and (5) be published from 1988 onwards to ensure contemporary data. We excluded intervention studies. We first screened titles and abstracts and included studies related to both polypharmacy and multimorbidity. However, during subsequent full-text screening, we excluded studies that did not specifically address polypharmacy or studies focusing on patients with a single disease $(n=9)$ or patients in palliative care $(n=1)$ as they are unlikely to reflect the experiences in relation to multimorbidity and polypharmacy in the broader population. Two authors independently carried out both rounds of screening and resolved any disagreements by consensus.

For articles that did not clarify whether patients took four or more medications, we contacted the corresponding author; one study was excluded after we received no reply. We used Covidence systematic review software (Melbourne, Australia). The Preferred Reporting Items for Systematic Reviews and Meta-Analyses flow diagram was used for reporting the study selection process. ${ }^{22}$

\section{Assessment of methodological quality}

We assessed the methodological quality of the included studies with the Critical Appraisal Skills Programme (CASP) qualitative checklist, designed to help researchers make sense of qualitative research in a systematic way. ${ }^{23}$ The 10-item checklist considers the validity of the results, the findings and their value in local settings. ${ }^{23}$ Two authors applied the checklist to included studies and resolved disagreements by consensus. No studies were excluded based on methodological quality.

\section{Data extraction}

We extracted data from each included study on authors, year, title, aim, study design, context, participants and analytical approach. Each finding, defined as the authors' interpretation of their results, ${ }^{24}$ was identified and coded by a single author and entered into a spreadsheet (Microsoft Excel, Redmond, Washington, USA) for all studies. If studies included other views than those of polypharmacy patients, only the data relating to patients' views were included in the synthesis. Using the terminology employed in the Joanna Briggs Institute Reviewer's Manual, each finding was assigned a level of credibility based on illustrations in the form of supporting quotations or field observations. ${ }^{24}$ Possible credibility levels were unequivocal (the illustration supported the finding beyond a reasonable doubt), credible (some association was present between the illustration and the finding, but it did not clearly support it, meaning that the finding was open to challenge) and unsupported (no illustration was presented). The credibility levels were assigned by CUE.

\section{Data synthesis}

We used meta-aggregation to synthesise the data. ${ }^{24}$ In this process, findings as expressed by researchers are 
summarised to produce generalisations that provide a basis for identifying recommendations for action. ${ }^{25}$ All findings are linked to a specific category and then to a specific synthesis, providing transparency in reporting. The endpoint of meta-aggregation is the formation of concrete recommendations for practice and research, increasing the practical utility of the synthesis. Specifically, the process of meta-aggregation includes three steps: (1) extracting all findings from the included articles, (2) developing categories of similar findings and (3) developing synthesised findings from at least two categories. ${ }^{25}$ Two authors, CUE and JTL, collaboratively derived categories and syntheses by inductively grouping similar findings and subsequently identifying conceptual categories. All syntheses, as well as the recommendations for practice and research that were subsequently developed, were discussed with all authors.
We report the data synthesis using the ENTREQ (Enhancing Transparency in Reporting the Synthesis of Qualitative Research) statement, ${ }^{26}$ an example of meta-aggregation, ${ }^{25}$ and the Joanna Briggs Institute Reviewer's Manual. ${ }^{27}$

\section{Patient and public involvement}

Neither patients nor the public were involved in the research process.

\section{RESULTS}

\section{Study inclusion}

The search strategy yielded 1676 citations (figure 1); 1305 remained after we removed duplicates and applied publication data and language restrictions. After screening titles and abstracts, we included 69 studies, of which 10 met the eligibility criteria after full-text screening. The

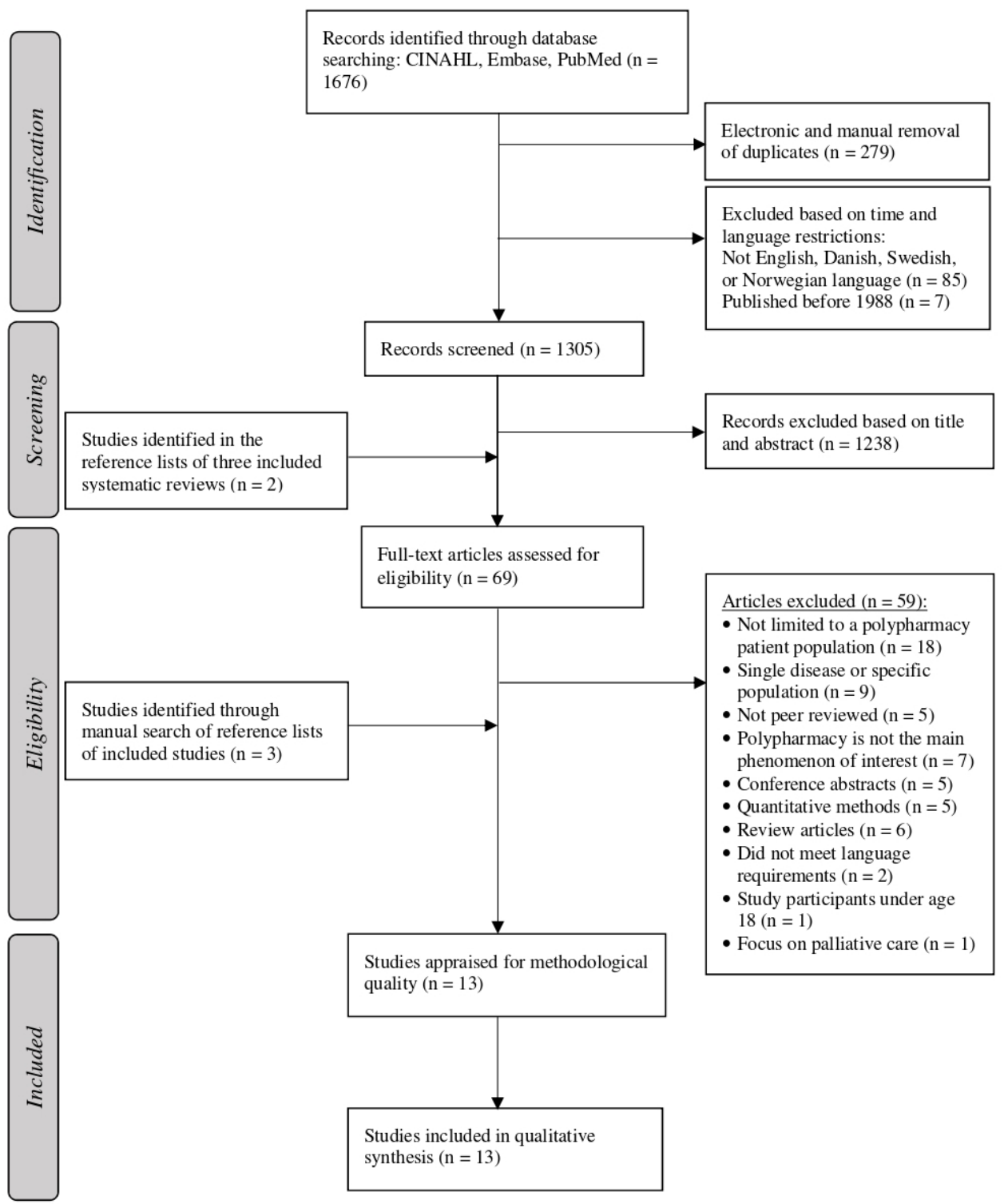

Figure 1 PRISMA flow diagram providing an overview of the selection process. CINAHL, Cumulative Index to Nursing and Allied Health Literature; PRISMA, Preferred Reporting Items for Systematic Reviews and Meta-Analyses. 
primary reasons for exclusion during full-text screening were study populations that were not limited to patients with polypharmacy $(n=18)$ and focus on a population with a specific disease $(n=9)$ or palliative care patients $(n=1)$. We identified three additional studies from reference lists, thus assessing a total of 13 studies for methodological quality and including them in the synthesis. ${ }^{28-40}$ All studies were reported in the English language. See online supplementary table 2 for a list of studies excluded during full-text screening.

\section{Study characteristics}

Eleven studies used semistructured individual and/ or focus group interviews, and two studies applied an unstructured approach to conducting individual interviews. Patients were recruited from primary care and community settings. A total of 499 patients taking four or more medications for varying chronic conditions were included, the majority of whom were over the age of 65 years and residing in communities. Five studies took place in the USA, three in the UK, three in Sweden, and one each in Australia and Germany (table 1).

\section{Methodological quality}

All 13 studies reported at least 8 of the 10 CASP checklist items. No studies met the criteria of adequately considering the relationship between researchers and participants, and only two studies ${ }^{31} 36$ failed to report the information required by all remaining CASP items (table 2).

\section{Synthesis}

We extracted 140 findings from the 13 studies, grouping them into 17 categories describing a wide variety of patient experiences of polypharmacy (online supplementary tables 3-7). We subsequently summarised the 17 categories into five interrelated syntheses describing central aspects of medication-related experiences of patients with polypharmacy (figure 2). For additional data supporting the syntheses, see online supplementary table 8 .

\section{Synthesis 1: patients with polypharmacy are a heterogeneous} group in terms of needing and appraising medication information Even though it is generally difficult for patients with polypharmacy to understand information about harms and benefits of medications, patients with polypharmacy are a heterogeneous group in terms of needing and appraising information and respond differently to the same information. These differing needs for and ways of responding to information are illustrated by Krska and colleagues ${ }^{31}$ :

More than half the participants felt they needed information to allay their concerns, beyond that provided directly by health professionals, which they obtained by various means, including books, patient information leaflets (PILs) and the internet. Conversely, four participants either did not want to know about their medicines or would never seek further information. One was uncertain about the usefulness of PILs and three considered that information caused or worsened rather than allayed concerns, meaning further reassurance and clarification from a health professional was needed.

Synthesis 2: patients are aware of the importance of medication adherence, but it is difficult to achieve

Patients are aware of the importance of adherence and of developing routines for taking medications. However, doing so requires substantial effort due to practical issues, regimen changes, and skipping, altering or forgetting doses, which may occur when patients assign different priorities to medications. Health professionals and patients' social networks can facilitate medication adherence. Some of the challenges to maintaining medication routines are illustrated by Krska and colleagues ${ }^{31}$ :

Although all participants had developed routines for using medicines, which had become automatic or 'second nature' - 'you think nothing of it' - interviews revealed the extent of the physical and organisational effort required by some patients in using medicines. ... The impact of changes in daily routine sometimes resulted in medicines not being used.

\section{Synthesis 3: decision-making about medications is complex}

Generally, patients want to take fewer medications. A multitude of factors affect patients' decision-making about medications, but physical function and the preference for a stable regimen are central. The desire for a stable regimen can even impact patients' willingness to discuss deprescribing. The following quotation from a participant in the study by Linsky and colleagues $^{32}$ illustrates the importance to patients of a stable regimen:

Oh, let's give it a time frame and find out how it goes. I'm not going to quit it completely because I already seen where my blood pressure was up there and sometimes I felt weird because I didn't even know what it was, and it was the high blood pressure. But if they are going to do something like that, well, if you want to do it on a trial basis and monitor me, fine, but right now it's got everything working fine. I'm not going to fool with something that's working good.

\section{Synthesis 4: multiple relational factors affect communication} between patients and physicians, and these factors can prevent patients from disclosing important information

Multiple factors in relationships between patients and healthcare professionals, such as unequal power relations, trust or lack thereof, problems in interacting with providers or in receiving care, and conflicting advice, affect communication and increase the risk that patients will not disclose important information about their 


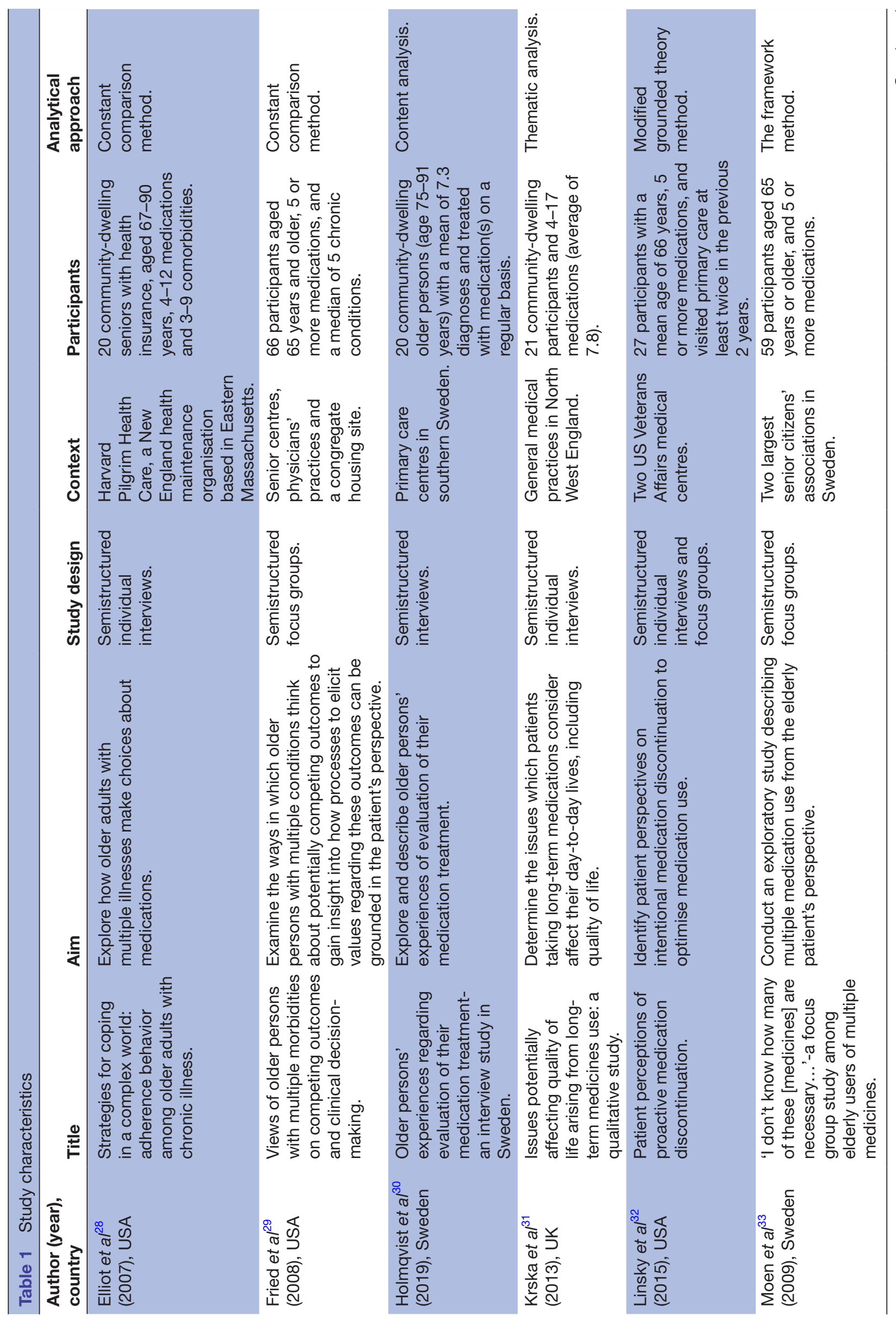




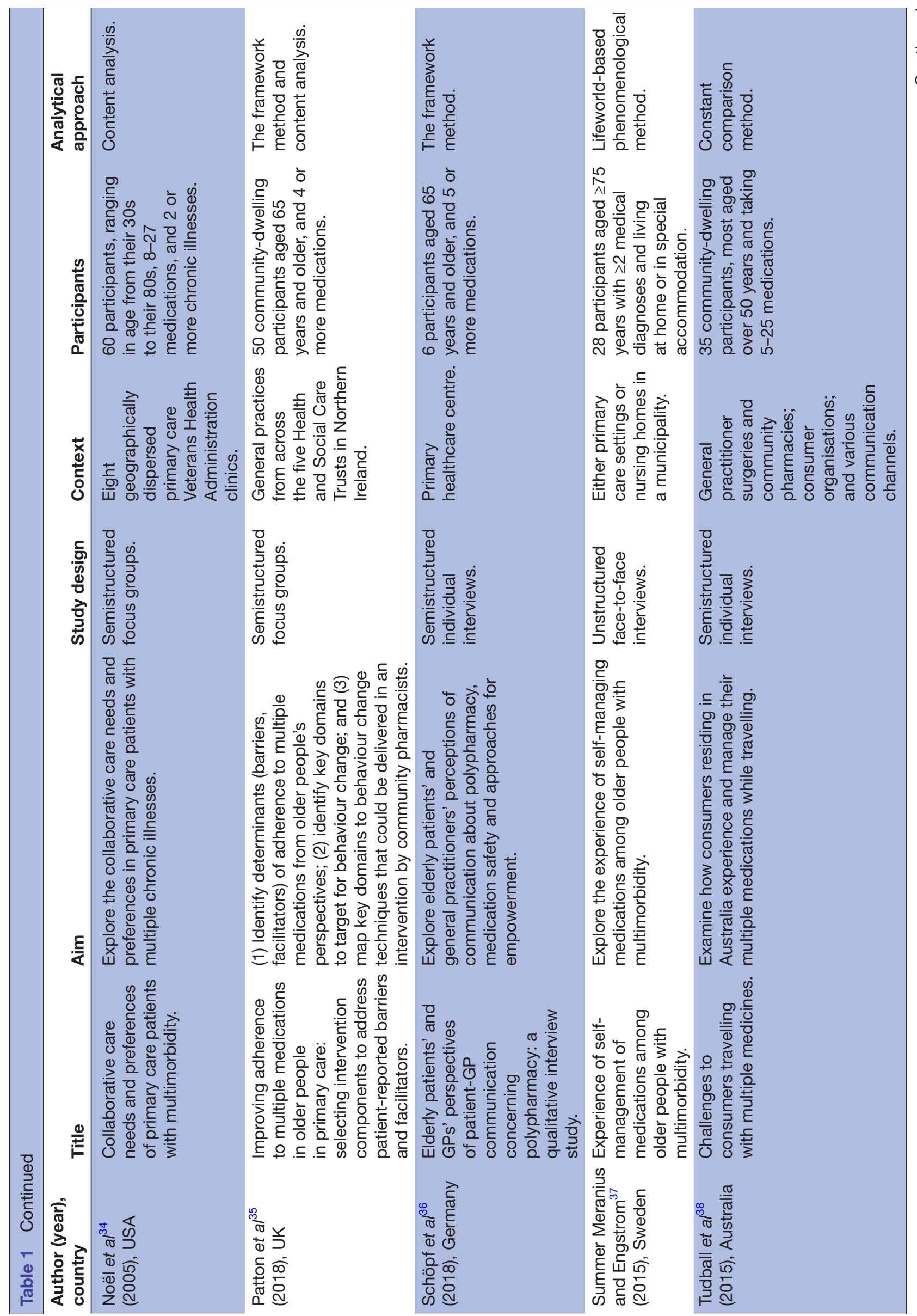




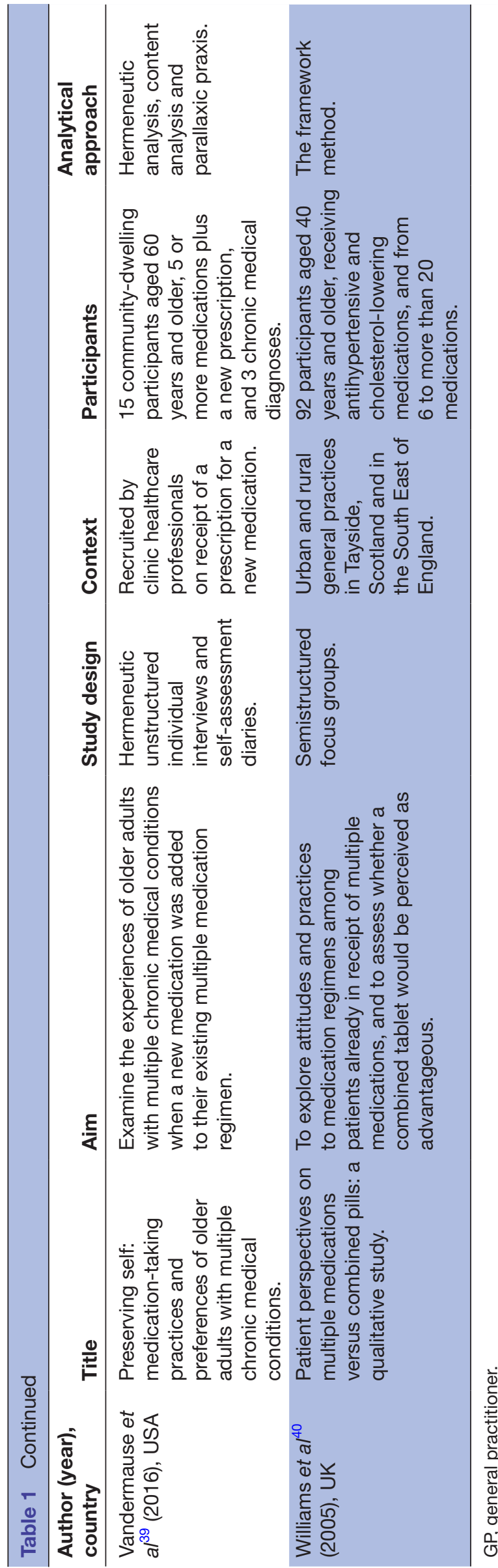

medication regimens. Continuity of care is important. Making changes to regimens without consulting prescribers beforehand is expressed in the following interpretation by Elliot and colleagues ${ }^{28}$ :

Experimenting with regimens ranged from stopping a medicine altogether; taking regular breaks; discontinuing medicines to check if they are working, or to determine the cause of side effects, trying individual medicines in a complex new regimen; and reducing doses. One patient reported stopping taking their antihypertensives to increase symptoms before a consultation. Prescribers were often not consulted before interviewees changed regimens.

Synthesis 5: polypharmacy affects patients' lives and selfperception, and challenges with polypharmacy are not limited to practical issues

The burden of polypharmacy does not arise solely from logistical challenges of organising medications and routines. Polypharmacy affects the self-perception of patients, can be seen by patients as embarrassing, and causes adverse effects that can have grave implications for patients' lives and affect their attitudes towards medications. The effect of taking multiple medications on patients' self-perception is illustrated by Vandermause and colleagues ${ }^{39}$ :

Participants did not want to fall into the category of 'taking multiple medicines.' Many rejected this descriptor, though they fit the criterion of taking five or more medicines. No matter what their medicines, they did not want to be seen as medication users. The desire to be viewed outside this designation was commonly strong. It was not uncommon to hear stories of confusion and dejection when participants described medicine side effects or compatibility problems, barriers to getting their prescriptions filled, and cost factors that were worrisome. All of these impediments affected their sense of who they were as persons. Their bodies were failing them, unless they could find a way to manage the problems they encountered.

\section{Proposed implications}

Based on the five syntheses, we proposed the following implications for practice:

- Healthcare professionals should actively solicit individual patients' perspectives on challenges related to polypharmacy (syntheses 1-5).

- Healthcare professionals should consider upscaling their communicative efforts to better inform and involve polypharmacy patients on their medications to an extent that reflects their needs (syntheses 1-5).

- Healthcare professionals should consider supporting patients with polypharmacy, preferably by involving patients' social network, by coordinating and ensuring continuity of care, as well as to facilitate dealing with 
Table 2 Results of the quality appraisal using the CASP checklist for qualitative studies

\begin{tabular}{|c|c|c|}
\hline Item & $\begin{array}{l}\text { Studies reporting the } \\
\text { item, } \mathrm{n}(\%)\end{array}$ & References \\
\hline Was there a clear statement of the aims of the research? & $13(100)$ & $28-40$ \\
\hline Was the research design appropriate to address the aims of the research? & $10(76.9)$ & $282931-3538-40$ \\
\hline Was the recruitment strategy appropriate to the aims of the research? & $12(92.3)$ & 28-30 32-40 \\
\hline Have ethical issues been taken into consideration? & $12(92.3)$ & $28-3537-40$ \\
\hline Was the data analysis sufficiently rigorous? & $12(92.3)$ & $28-3032-40$ \\
\hline Is there a clear statement of findings? & $13(100)$ & $28-40$ \\
\hline
\end{tabular}

CASP, Critical Appraisal Skills Programme.

practical and psychological issues with taking many medications (syntheses 2, 4 and 5).

In addition, we propose the following recommendations for strengthening research in medication-related experiences of multimorbid patients with polypharmacy:

- Studies of the different medication information needs of patients with polypharmacy should be conducted (synthesis 1).

- Interventions promoting continuity of care should be evaluated qualitatively to assess patients' perspectives on polypharmacy (synthesis 4).

\section{DISCUSSION}

To the best of our knowledge, this is the first systematic review of qualitative studies to comprehensively compile medication-related experiences of multimorbid patients with polypharmacy. From the 13 included studies, we extracted a plethora of experiences of being a patient with polypharmacy and formed five interrelated syntheses related to the differing medication information needs, the difficulties of achieving adherence, the complexity of decision-making, the relationship between healthcare professionals and patients, and the impact on patients' self-perception. These formed the basis for several recommendations that can improve care for patients with polypharmacy, as well as guide future research in medication-related experiences of multimorbid patients with polypharmacy.

One important aspect of synthesis 2 is the potential facilitating role that patients' social network can play in medication adherence. This finding of social support being important to ensuring proper medication use is consistent with previous findings. ${ }^{10}$ Also, experiencing a lack of understanding from others about one's condition negatively affected patients' beliefs about medications. ${ }^{10}$ This calls for healthcare professionals to include patients' support networks in consultations or communicate with friends and relatives providing social support to ensure they understand the necessity for treatment and consequences of non-adherence.

A finding central to our synthesis is the challenges that patients with polypharmacy experience in the relationship with healthcare professionals. These relationships are challenged by multiple factors, such as power imbalances, varying levels of trust, problems in interacting with providers or in receiving care, and conflicting advice. Schiøtz et $a l^{13}$ reported that patients with polypharmacy in a Danish context generally trusted healthcare professionals. In general, this finding was reflected in the included studies. However, blind trust may prevent patients from asking questions and disclosing important information relevant to their condition. Rather than focusing on establishing trust between patients and healthcare professionals as a means of improving adherence, it may be more important to address power imbalances and to increase continuity of care for patients, particularly relational continuity, defined as 'an ongoing therapeutic relationship between a patient and one or more providers'. ${ }^{41}$ Mohammed et $a l^{10}$ found a link between the lack of an established relationship with healthcare professionals and both inappropriate medication-related behaviour and a negative attitude towards treatment. A larger treatment burden and more complex regimen involving multiple healthcare professionals may increase the risk that patients have no established patient-healthcare professional relationship. In addition, Mohammed et $a l^{10}$ argue that healthcare professionals should direct their attention towards patients' lived experiences with medications; a focus on patients' medication-related needs can lead to improvements in medication therapy and health outcomes. Thus, healthcare professionals must focus on medication-related experiences of patients with polypharmacy to address experiences or attitudes that can affect medication adherence. In a qualitative study of barriers to providing medication reviews for multimorbid 


\section{Synthesis 1}

Even though it is generally difficult for patients with polypharmacy to understand information about harms and benefits of medications, patients with polypharmacy are a heterogeneous group in terms of needing and appraising information and respond differently to the same information.

\section{Synthesis 2}

Patients are aware of the importance of adherence and of developing routines for taking medications. However, doing so requires substantial effort due to practical issues, regimen changes, and skipping, altering, or forgetting doses, which may occur when patients assign different priorities to medications.

Health professionals and patients' social networks can facilitate medication adherence.
- Category 1: It is hard for patients with polypharmacy to understand and balance harms and benefits of medications.

- Category 2: Patients with polypharmacy have different needs for information. However, patients need health professionals to provide information when regimens are changed.
- Category 3: Adherence is highly affected by practical issues.

- Category 4: Routines are critical but can be difficult to maintain. Support from aids or reminders, family, and/or healthcare professionals can facilitate adherence.

- Category 5: All medications are not equally important to patients.

- Category 6: Patients are aware of the importance of adherence, yet deliberately skip or alter and forget doses.

\section{Synthesis 3}

Generally, patients want to take fewer medications. A multitude of factors affect patients' decision-making about medications, but physical function and the preference for a stable regimen are central. The desire for a stable regimen can even impact patients' willingness to discuss deprescribing.
- Category 7: Various factors affect decision-making about medications, even though a single factor is often dominant. Maintaining or improving physical function appears to be the most central treatment goal for patients above other factors, such as extending life.

- Category 8: Patients want to reduce the number of medications, while also wanting the beneficial effects of medications.

- Category 9: A stable regimen is important to patients, and this sometimes reduces their willingness to have their medication regimen altered and to even discuss deprescribing with prescribers. However, this can be a result of the medications working well for the patient.

\section{Synthesis 4}

Multiple factors in relationships between patients and healthcare professionals, such as unequal power relations, trust or lack thereof, problems in interacting with providers or in receiving care, and conflicting advice, affect communication and increase the risk that patients will not disclose important information about their medication regimens. Continuity of care is important.
- Category 10: The healthcare professional, the doctor in particular, is very important to patients, but trust in doctors can also hinder open communication, by patients keeping from asking about important information.

- Category 11: The unequal power dynamics between patients and doctors can affect communication. Some patients compensate by preparing before a consultation, for example by seeking information.

- Category 12: Patients make changes to their regimens without disclosing it to healthcare professionals. Withholding information can be related to the relationship with the healthcare professional.

- Category 13: Physicians provide conflicting advice to patients.

- Category 14: Patients experience problems in interacting with providers or in receiving care, and insufficient time with healthcare professionals when seeking care. Continuity of care is important to patients.

\section{Synthesis 5}

The burden of polypharmacy does not arise solely from logistical challenges of organising medications and routines. Polypharmacy affects the self-perception of patients, can be seen by patients as embarrassing, and causes adverse effects that can have grave implications for patients' lives and affect their attitudes toward medications.
- Category 15: Medication-taking is a disruptive burden affecting patients' lives and limiting spontaneity. Not having tangible effects of medications can lead to hopelessness.

- Category 16: Being a multiple-medication user can be seen by patients as embarrassing.

- Category 17: Adverse effects have grave implications on patients' lives and attitudes toward medications, and these might at times not be taken seriously by healthcare professionals.

Figure 2 Syntheses. The figure provides an overview of the five syntheses, along with the categories from which they are formed. 
patients with polypharmacy, general practitioners (GPs) expressed a clear need to improve information exchange between sectors by, for example, establishing a dialogue and collaboration between GPs and specialists. ${ }^{42}$ This pertains to the current study in that information exchange between sectors could improve the relationship between patients and healthcare professionals and, in particular, improve patients' general perceptions of the healthcare system as a unified entity in which information is consistent and shared across sectors.

The finding from synthesis 5 of the impact of polypharmacy on self-perception expands on previous findings. In a synthesis of qualitative studies of medication-taking in general, certain medications, such as those for mental health conditions, were reported as being particularly stigmatised, likely due to the stigma attached to mental illness. ${ }^{43}$ Our syntheses expand on this by suggesting that taking multiple medications, regardless of underlying conditions, can be perceived as embarrassing by patients. This feeling can affect how patients perceive and make decisions about taking medications and may affect adherence negatively. Therefore, we see a clear need to target the perception of medications among patients and their social network to increase the adherence and quality of life for patients with polypharmacy.

In assessing study strengths and limitations, four evaluation criteria for qualitative research are relevant: credibility, transferability, dependability and confirmability. ${ }^{44}$ The credibility of our categories and syntheses is enhanced by our triangulation of findings from different studies and transparent report. However, the transferability of our findings to all patients taking more than four medications is unknown. We included 13 studies with participants who varied in terms of the number and types of their medications and diagnoses and the healthcare systems in which they received care, which may limit transferability to some degree. However, our use of four or more medications as the definition of polypharmacy arose from our aim to capture a broad range of patient experiences of polypharmacy; this definition was applied in 4 of the 13 included studies. We did not use the most common definition of polypharmacy, ${ }^{2}$ but we believe our findings express common experiences of people with multimorbidity and polypharmacy. Our findings are dependable because we assessed the credibility of each finding from all papers; we are confident that our findings are supported by the original data. In addition, the transparency of our reporting (online supplemental file 1) allows the reader to assess how well our syntheses are supported by the findings. However, as is the case with all qualitative methods, the subjective nature of our analysis introduces a risk of bias. A factor potentially limiting the confirmability of our study is that the involved researchers influence the process of extracting and grouping data, and a single author completed data extraction. However, problems, doubts and unclear texts were discussed with the author group, and two authors collaborated in the data synthesis. Other factors mitigating bias include transparency in reporting, triangulation and assessing our findings in light of what was previously known. Additional strengths are our stringent process of selecting and appraising studies with a preplanned and structured approach to searching and appraising literature, the use of two reviewers for the screening and quality appraisal, and the use of CASP checklist for assessing the methodological quality of included studies.

\section{CONCLUSION}

Polypharmacy has a range of consequences and poses many challenges to patients. We identified five syntheses emphasising themes of central importance for patients with multimorbidity and polypharmacy relating to medication information needs, adherence, decision-making, the relationship between healthcare professionals and patients, and patients' self-perception. These themes can affect adherence and quality of life, and it is therefore essential that healthcare professionals actively solicit individual patients' perspectives on challenges related to polypharmacy. Based on the reported experiences, we recommend that healthcare professionals upscale communicative efforts and involvement of patients' social network on an individualised basis to facilitate shared decision-making and treatment adherence in many patients with multimorbidity and polypharmacy.

\section{Author affiliations}

${ }^{1}$ Center for Clinical Research and Prevention, Bispebjerg and Frederiksberg Hospital, Capital Region of Denmark, Frederiksberg, Denmark

${ }^{2}$ The Research Unit for General Practice, Aarhus, Denmark

${ }^{3}$ Research Group for Social and Clinical Pharmacy, Department of Pharmacy,

University of Copenhagen, Faculty of Health and Medical Sciences, Copenhagen, Denmark

${ }^{4}$ Global Business Quality Management, Falck, Copenhagen, Denmark

${ }^{5}$ Department of Clinical Pharmacology, Bispebjerg and Frederiksberg Hospital, University of Copenhagen, Faculty of Health and Medical Sciences, Copenhagen, Denmark

${ }^{6}$ The Research Unit for General Practice, Department of Public Health, University of Copenhagen, Faculty of Health and Medical Sciences, Copenhagen, Denmark ${ }^{7}$ Innovation and Research Center for Multimorbidity and Chronic Conditions, Region Zealand, Slagelse, Denmark

Contributors CUE is the guarantor of the submitted work. CUE planned the searches in collaboration with RJ, JTL, MBC and AF; conducted the searches; carried out study selection in collaboration with SK; assessed the methodological quality in collaboration with LDC; extracted the findings; synthesised the findings in collaboration with JTL; and wrote the manuscript. CUE, RJ, JTL, MBC and AF devised the project and provided input for the synthesis of findings. All authors critically revised the manuscript. Data synthesis was conducted by CUE with expertise in literature review and JTL with extensive experience in qualitative research on patient and professional perspectives on use of medications.

Funding CUE and SK were funded by a grant from the Capital Region of Denmark. No funding body has influenced the study design; data collection, analysis and interpretation of data; writing of the report; and in the decision to submit the article for publication.

Patient consent for publication Not required.

Ethics approval The study did not require ethical approval.

Provenance and peer review Not commissioned; externally peer reviewed.

Data availability statement Data are available upon reasonable request from the corresponding author. 
Open access This is an open access article distributed in accordance with the Creative Commons Attribution Non Commercial (CC BY-NC 4.0) license, which permits others to distribute, remix, adapt, build upon this work non-commercially, and license their derivative works on different terms, provided the original work is properly cited, appropriate credit is given, any changes made indicated, and the use is non-commercial. See: http://creativecommons.org/licenses/by-nc/4.0/.

ORCID iD

Christian Ulrich Eriksen http://orcid.org/0000-0002-7163-5703

\section{REFERENCES}

1 Muth C, Glasziou PP. Guideline recommended treatments in complex patients with multimorbidity. BMJ 2015;351:h5145.

2 Masnoon N, Shakib S, Kalisch-Ellett L, et al. What is polypharmacy? A systematic review of definitions. BMC Geriatr 2017;17:230.

3 Cadogan CA, Ryan C, Hughes CM. Appropriate polypharmacy and medicine safety: when many is not too many. Drug Saf 2016;39:109-16.

4 Koper D, Kamenski G, Flamm M, et al. Frequency of medication errors in primary care patients with polypharmacy. Fam Pract 2013;30:313-9.

5 Schiøtz ML, Høst D, Christensen MB, et al. Quality of care for people with multimorbidity - a case series. BMC Health Serv Res 2017;17:745.

6 Michaelsen $\mathrm{MH}$, McCague P, Bradley CP, et al. Medication reconciliation at discharge from Hospital: a systematic review of the quantitative literature. Pharmacy 2015;3:53-71.

7 Belda-Rustarazo S, Cantero-Hinojosa J, Salmeron-García A, et al. Medication reconciliation at admission and discharge: an analysis of prevalence and associated risk factors. Int J Clin Pract 2015;69:1268-74.

8 Assiri GA, Shebl NA, Mahmoud MA, et al. What is the epidemiology of medication errors, error-related adverse events and risk factors for errors in adults managed in community care contexts? A systematic review of the international literature. BMJ Open 2018;8:e019101.

9 Pasina L, Brucato AL, Falcone C, et al. Medication nonadherence among elderly patients newly discharged and receiving polypharmacy. Drugs Aging 2014;31:283-9.

10 Mohammed MA, Moles RJ, Chen TF. Medication-Related burden and patients' lived experience with medicine: a systematic review and metasynthesis of qualitative studies. BMJ Open 2016;6:e010035.

11 Reeve E, Wolff JL, Skehan M, et al. Assessment of attitudes toward deprescribing in older Medicare beneficiaries in the United States. JAMA Intern Med 2018;178:1673-80.

12 Aoki T, Yamamoto Y, Ikenoue T, et al. Factors associated with patient preferences towards deprescribing: a survey of adult patients on prescribed medications. Int J Clin Pharm 2019;41:531-7.

13 Schiøtz ML, Frølich A, Jensen AK, et al. Polypharmacy and medication deprescribing: a survey among multimorbid older adults in Denmark. Pharmacol Res Perspect 2018;6:e00431-e31.

14 Galazzi A, Lusignani M, Chiarelli MT, et al. Attitudes towards polypharmacy and medication withdrawal among older inpatients in Italy. Int J Clin Pharm 2016;38:454-61.

15 Kua KP, Saw PS, Lee SWH. Attitudes towards deprescribing among multi-ethnic community-dwelling older patients and caregivers in Malaysia: a cross-sectional questionnaire study. Int J Clin Pharm 2019;41:793-803.

16 Tegegn HG, Tefera YG, Erku DA, et al. Older patients' perception of deprescribing in resource-limited settings: a cross-sectional study in an Ethiopia university hospital. BMJ Open 2018;8:e020590.

17 Reeve E, Low L-F, Hilmer SN. Attitudes of older adults and caregivers in Australia toward deprescribing. J Am Geriatr Soc 2019;67:1204-10.

18 Rankin A, Cadogan CA, Patterson SM, et al. Interventions to improve the appropriate use of polypharmacy for older people. Cochrane Database Syst Rev 2018;9:CD008165.

19 Cipolle R, Strand L, Morley P. Chapter 4. Patient-Centeredness in Pharmaceutical Care. In: Pharmaceutical Care Practice:The patientcentered approach to medication management services. Third ed. McGraw-Hill Education / Medical, 2012.
20 Malterud K. Kvalitativ metasyntese som forskningsmetode i medisin og helsefag [Qualitative metasynthesis as a research method in medical and health sciences]. Universitetsforlaget, 2017.

21 Booth A. Searching for qualitative research for inclusion in systematic reviews: a structured methodological review. Syst Rev 2016;5:74.

22 Moher D, Liberati A, Tetzlaff J, et al. Preferred reporting items for systematic reviews and meta-analyses: the PRISMA statement. PLoS Med 2009;6:e1000097.

23 Critical Appraisal Skills Programme. CASP Qualitative Checklist. Available: https://casp-uk.net/wp-content/uploads/2018/01/CASPQualitative-Checklist-2018.pdf [Accessed 23 Aprl 2018].

24 Aromataris E, Munn Z, eds. Joanna Briggs Institute Reviewer's Manual. The Joanna Briggs Institute, 2017.

25 Hannes K, Pearson A. Obstacles to the Implementation of EvidenceBased Practice in Belgium: A Worked Example of Meta-Aggregation. In: Lockwood C, ed. Synthesizing Qualitative Research, 2012.

26 Tong A, Flemming K, Mclnnes E, et al. Enhancing transparency in reporting the synthesis of qualitative research: ENTREQ. BMC Med Res Methodol 2012;12:181.

27 Lockwood C, Porrit K. Chapter 2: Systematic reviews of qualitative evidence. In: Munn Z, Aromataris E, eds. JBI Manual for Evidence Synthesis. The Joanna Briggs Institute, 2017.

28 Elliott RA, Ross-Degnan D, Adams AS, et al. Strategies for coping in a complex world: adherence behavior among older adults with chronic illness. J Gen Intern Med 2007;22:805-10.

29 Fried TR, McGraw S, Agostini JV, et al. Views of older persons with multiple morbidities on competing outcomes and clinical decisionmaking. J Am Geriatr Soc 2008;56:1839-44.

30 Holmqvist M, Thor J, Ros A, et al. Older persons' experiences regarding evaluation of their medication treatment-An interview study in Sweden. Health Expect 2019;22:1294-303.

31 Krska J, Morecroft CW, Poole H, et al. Issues potentially affecting quality of life arising from long-term medicines use: a qualitative study. Int J Clin Pharm 2013;35:1161-9.

32 Linsky A, Simon SR, Bokhour B. Patient perceptions of proactive medication discontinuation. Patient Educ Couns 2015;98:220-5.

33 Moen J, Bohm A, Tillenius T, et al. 'I don't know how many of these [medicines] are necessary...'- a focus group study among elderly users of multiple medicines. Patient Educ Couns 2009;74:135-41.

34 Noël PH, Frueh BC, Larme AC, et al. Collaborative care needs and preferences of primary care patients with multimorbidity. Health Expect 2005;8:54-63.

35 Patton DE, Cadogan CA, Ryan C, et al. Improving adherence to multiple medications in older people in primary care: selecting intervention components to address patient-reported barriers and facilitators. Health Expect 2018;21:138-48.

36 Schöpf AC, von Hirschhausen M, Farin E, et al. Elderly patients' and GPs' perspectives of patient-GP communication concerning polypharmacy: a qualitative interview study. Prim Health Care Res Dev 2018;19:355-64.

37 Summer Meranius M, Engstrom G. Experience of self-management of medications among older people with multimorbidity. J Clin Nurs 2015;24:2757-64.

38 Tudball J, Smith L, Ryan K, et al. Challenges to consumers travelling with multiple medicines. J Pharm Health Serv Res 2015;6:127-32.

39 Vandermause R, Neumiller JJ, Gates BJ, et al. Preserving Self: Medication-Taking Practices and Preferences of Older Adults with Multiple Chronic Medical Conditions. J Nurs Scholarsh 2016;48:533-42.

40 Williams B, Shaw A, Durrant R, et al. Patient perspectives on multiple medications versus combined pills: a qualitative study. QJM 2005;98:885-93.

41 Haggerty JL, Reid RJ, Freeman GK, et al. Continuity of care: a multidisciplinary review. BMJ 2003;327:1219-21.

42 Laursen J, Kornholt J, Betzer C, et al. General Practitioners' Barriers Toward Medication Reviews in Polymedicated Multimorbid Patients: How Can a Focus on the Pharmacotherapy in an Outpatient Clinic Support GPs? Health Serv Res Manag Epidemiol 2018;5:2333392818792169.

43 Pound P, Britten N, Morgan M, et al. Resisting medicines: a synthesis of qualitative studies of medicine taking. Soc Sci Med 2005;61:133-55.

44 Lincoln YS, Guba EG, Pilotta JJ. Naturalistic inquiry. Newbury Park, CA: Sage Publications, 1985: 9. 438-9. 